\title{
Effects of different concentrations of type-I collagen hydrogel on the growth and differentiation of chondrocytes
}

\author{
DESHAN HU and XIULI SHAN \\ Department of Stomatology, The Fifth People's Hospital of Jinan, Jinan, Shandong 250022, P.R. China
}

Received May 15, 2017; Accepted September 19, 2017

DOI: $10.3892 /$ etm.2017.5202

\begin{abstract}
The objective of this study was to analyze the effects of type-I collagen hydrogel of different concentrations on the growth and differentiation of rabbit chondrocytes. Articular cartilage from New Zealand white rabbits was harvested and cultured. Second-generation chondrocytes were collected for in vitro culture with 10,7 , and $5 \mathrm{mg} / \mathrm{ml}$ type-I collagen hydrogel, respectively (denoted as groups A, B, and C). After in vitro culture for 1 day, chondrocytes were stained with fluorescein diacetate (FDA)/propidium iodide (PI), and cell viability was observed by laser confocal microscopy. After in vitro culture for 14 days, the histological patterns were observed by H\&E and toluidine blue staining. The expression of chondrocyte-related genes were measured by real-time quantitative RT-PCR. After in vitro culture for 1 day, FDA/PI staining showed that the cell density of group A was significantly higher than that of group B and C. After in vitro culture for 14 days, H\&E staining showed that chondrocytes showed obvious aggregation in group A, partial proliferation and aggregation in group B, and uniform distribution in group $\mathrm{C}$. Toluidine blue staining showed that chondrocytes in group A had aggregation areas and some were stained purple-red, fewer chondrocytes were aggregated with different staining around them in group B, and the aggregation of chondrocytes was not obvious. However, the distribution of chondrocytes was uniform with different staining in group $\mathrm{C}$. After in vitro culture for 2 weeks, the levels of polymerized proteoglycan and type-II collagen mRNA were not significantly different between the three groups $(\mathrm{P}>0.05)$. The levels of type-I collagen, type-X collagen, and Sox 9 mRNA in group A were significantly higher than those in group $\mathrm{B}$ and $\mathrm{C}(\mathrm{P}<0.05)$. In conclusion, high concentration type-I collagen hydrogel can promote chondrocyte fibrosis and upregulation of type-I collagen, type-X collagen, and Sox 9 mRNA.
\end{abstract}

Correspondence to: Dr Deshan Hu, Department of Stomatology, The Fifth People's Hospital of Jinan, 24297 Jingshi Road, Jinan, Shandong 250022, P.R. China

E-mail: drdeshanhu@163.com

Key words: type-I collagen hydrogel, chondrocyte, Sox9

\section{Introduction}

Articular cartilage injury is a common clinical orthopedic disease. Self-repair of articular cartilage is difficult at pathogenic sites, and there are no specific methods to repair articular cartilage injury in clinical practice (1). In recent years, with rapid developments in modern biology and advanced materials, the application of cartilage tissue engineering has promoted the emergence of new methods to repair articular cartilage injury (2). Articular cartilage is rich in type-II collagen, and the synthesis and secretion of type-II collagen are specific indicators of articular chondrocytes to maintain their differentiated phenotype. In addition, they are essential bases for the evaluation of cartilage tissue engineering (3). In cartilage tissue engineering, commonly used accelerants include growth factors, such as exogenous growth factors, which are expensive and can easily biodegrade. Moreover, they may have immunogenicity and other problems, limiting their clinical application (4). Therefore, it is necessary to actively explore more effective accelerants with improved bio-performance. Type-I collagen is widely distributed. Studies have confirmed that (5) type-I collagen has good biocompatibility and biodegradability, and has been used in hemostasis, and in artificial skin and corneal repair. Previous studies showed that (6) type-I collagen scaffolds have good prospects in cartilage tissue engineering and cartilage repair. However, there are few studies on the effects of type-I collagen hydrogel of different concentrations on chondrocyte growth and differentiation. To further optimize the concentration of type-I collagen in collagen hydrogel, its effects on articular chondrocytes from New Zealand white rabbits were analyzed.

\section{Materials and methods}

Experimental animals. Three newborn New Zealand white rabbits (male or female) aged 1 week were selected. The average weight was $0.17 \mathrm{~kg}$. The rabbits were provided by the animal center of the Fifth People's Hospital of Jinan. The study was approved by Ethics Committee of the the Fifth People's Hospital of Jinan.

Reagents. Type-I collagen was extracted from calf skin, and prepared by the pathology laboratory of the Fifth People's Hospital of Jinan; $\alpha$-MEM medium (Shanghai Huiying Biotechnology Co., Ltd.); trypsin (Beijing Geyuan Tianrun 
Biotechnology Co., Ltd.); type-II collagenase (Shanghai Shize Biotechnology Co., Ltd.); fluorescein diacetate (FDA)/propidium iodide (PI) dye (Shanghai Hualan Chemical Technology Co., Ltd.); DNA extraction kit (Shenzhen Baoankang Biology Co., Ltd.); ReactTra Ace Qpcr RT kit [Toyobo (Shanghai) Biotechnology Co., Ltd.]; acetic acid, $\mathrm{NaOH}$, formaldehyde, and other chemical reagents were from Sinopharm Group Chemical Reagent Co., Ltd.

Preparation of collagen hydrogel. Appropriate amounts of type-I collagen and $0.5 \mathrm{~mol} / \mathrm{l}$ acetic acid aqueous solution were weighed and mixed. The $\mathrm{pH}$ of the solution was adjusted to approximately $7.5 \mathrm{using} 2 \mathrm{~mol} / \mathrm{l} \mathrm{NaOH}$ solution at $4^{\circ} \mathrm{C}$, and the final concentration of type-I collagen was $10 \mathrm{mg} / \mathrm{ml}$. The solution was then diluted to 7 and $5 \mathrm{mg} / \mathrm{ml}$, respectively. Approximately $0.5 \mathrm{ml}$ of collagen solutions of different concentrations were placed in a water bath at constant temperature $\left(37^{\circ} \mathrm{C}\right)$ for $30 \mathrm{~min}$ to prepare the collagen hydrogel with type-I collagen concentrations of 10,7 , and $5 \mathrm{mg} / \mathrm{ml}$.

Chondrocyte extraction and inoculation. Articular cartilages from three New Zealand white rabbits were harvested, and the chondrocytes were separated by enzyme digestion, and cultured. Chondrocytes were then collected, and appropriate amounts of $\alpha$-MEM medium were added to prepare cell suspensions. Cell suspensions were seeded in culture dishes at the density of $1 \times 10^{5}$ cells $/ \mathrm{ml}$, and then cultured in an illumination incubator. After cells were confluent, $0.25 \%$ trypsin was used for digestion, followed by subculture. The morphology of second-generation chondrocytes was observed under an inverted fluorescence phase-contrast microscope.

Second-generation chondrocytes were collected and cultured in vitro with 10,7 , and $5 \mathrm{mg} / \mathrm{ml}$ type-I collagen hydrogel, respectively. The above three sterile collagen solutions were mixed and the suspensions had a concentration of $5 \times 10^{6}$ cells $/ \mathrm{ml}$. The cell suspensions were then cultured in an incubator and added to the $300 \mu \mathrm{l}$ collagen hydrogel-chondrocyte complex after the collagen solution produced hydrogel, denoted as groups A, B, and C with 10, 7, and $5 \mathrm{mg} / \mathrm{ml}$ type-I collagen hydrogel, respectively. The complex continued to be cultured in an incubator for 2 weeks, and the solution was replaced every 2 days.

Observation of cell viability. The collagen hydrogel-chondrocyte complexes (one in each group) were selected after in vitro culture for 1 day, and stained with fluorescein diacetate (FDA)/propidium iodide (PI). Cell viability was observed under a laser confocal microscope.

Histological observation. The collagen hydrogel-chondrocyte complexes were selected after in vitro culture for 2 weeks and fixed using $10 \%$ neutral formaldehyde, followed by paraffin embedding and sectioning at $5 \mu \mathrm{m}$. Sections were then stained with $\mathrm{H} \& \mathrm{E}$ and toluidine blue to observe the cell distribution.

Real-time quantitative RT-PCR. The collagen hydrogelchondrocyte complexes were selected after in vitro culture for 2 weeks. Real-time quantitative RT-PCR was used to measure the mRNA levels of type-II collagen, polymerized proteoglycan, type-I collagen, type-X collagen, Sox9, and other

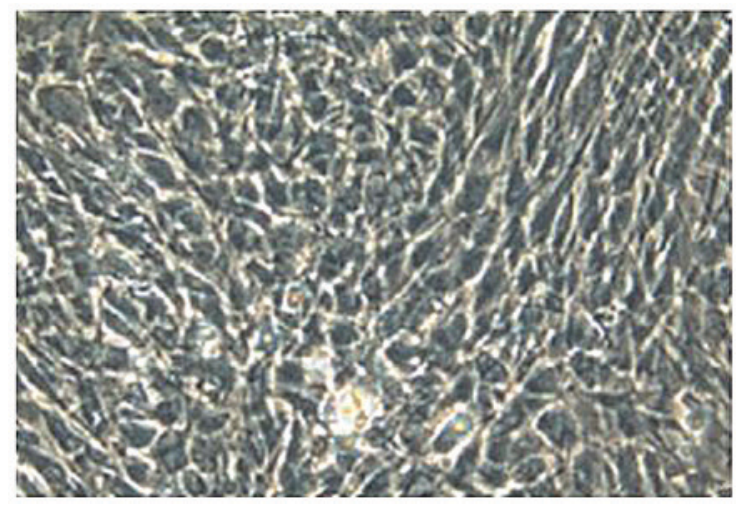

Figure 1. Morphological observation of second-generation chondrocytes after in vitro culture for 1 day. Inverted fluorescence phase-contrast microscopy showed that the cells were mostly polygonal with good growth status (x200).
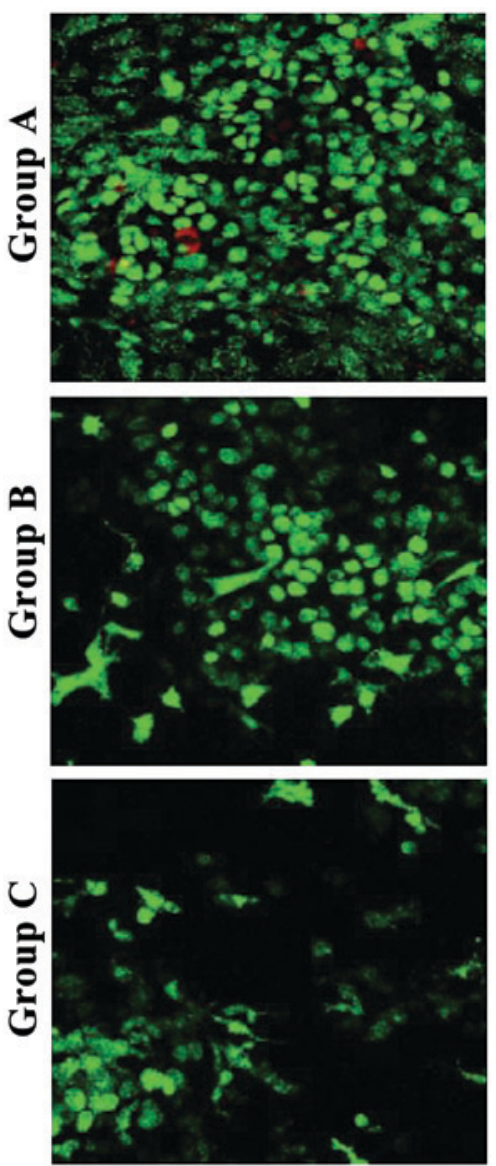

Figure 2. FDA/PI staining of collagen hydrogel-chondrocyte complexes after in vitro culture for 1 day in the three groups. Most of the chondrocytes in collagen hydrogels of groups A, B, and C were stained green, while a few were stained red (x200).

chondrocyte-related genes. The housekeeping gene, GAPDH, was used to normalize the expression of each gene. mRNA was extracted from samples using a specific extraction kit, followed by reverse transcription into cDNA using a ReverTra Ace Qpcr RT kit. The cDNA was then amplified using SsoFast ${ }^{\mathrm{TM}}$ EvaGreen ${ }^{\circledR}$ Supermix reagent (Beijing Changlitong Technology Co., Ltd.) in a CFX960 real-time fluorescence quantitative PCR instrument. The expression of each gene was analyzed, and each sample was measured three times. Related 

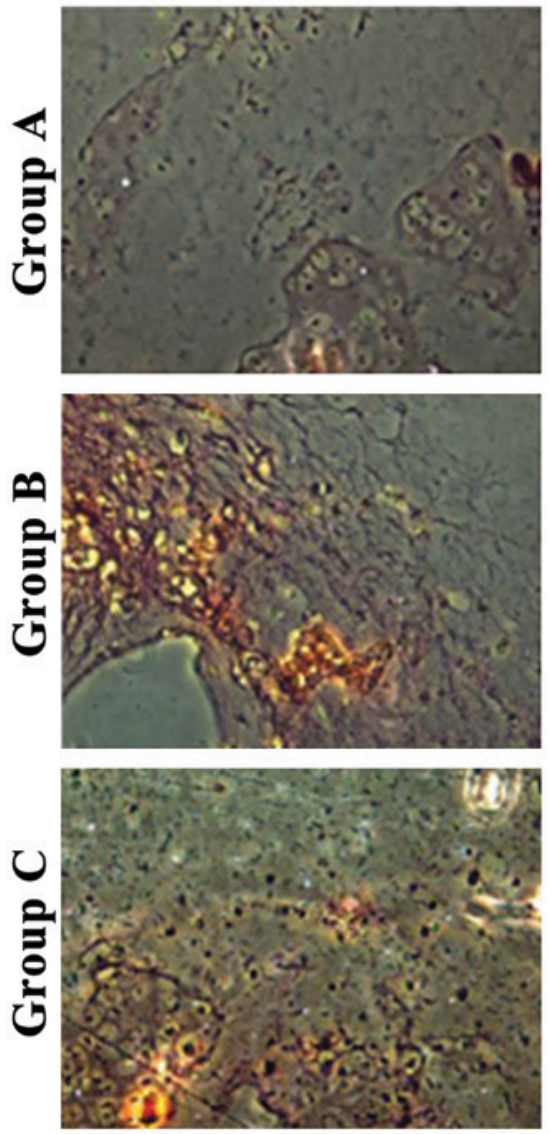

Figure 3. H\&E staining and observation of collagen hydrogel-chondrocyte complexes after in vitro culture for 2 weeks in the three groups. Chondrocytes were aggregated significantly in group A, some chondrocytes showed proliferation and aggregation in group $\mathrm{B}$, and the distribution of chondrocytes was relatively uniform in group C (x200).

primers were designed and synthesized by Shanghai Gongda Co., Ltd.

Statistical analysis. SPSS 20.0 (IBM, New York, NY, USA) statistical software was used for data analysis. Numerical data are presented as mean $\pm \mathrm{SD}$, and compared using the paired samples t-test. The Chi-square test was used for comparisons of numerical data. $\mathrm{P}<0.05$ was considered to indicate a statistically significant difference.

\section{Results}

Chondrocyte inoculation and observation of viability. Observation by inverted fluorescence phase-contrast microscopy showed that the second-generation chondrocytes were polygonal with diameter of approximately $8-10 \mu \mathrm{m}$, and had good growth status (Fig. 1). After collagen hydrogel-chondrocyte complexes in groups $\mathrm{A}, \mathrm{B}$, and $\mathrm{C}$ were cultured in vitro for 1 day, and stained with FDA/PI, laser confocal microscopy showed that the distribution of chondrocytes was uniform. Most were spherical, while some were polygonal in shape, with a tendency to proliferate and aggregate. Most of the chondrocytes in collagen hydrogels of groups A, B, and C were stained green, while a few were stained red. The cell density of group A was significantly higher than that of group B and C (Fig. 2).
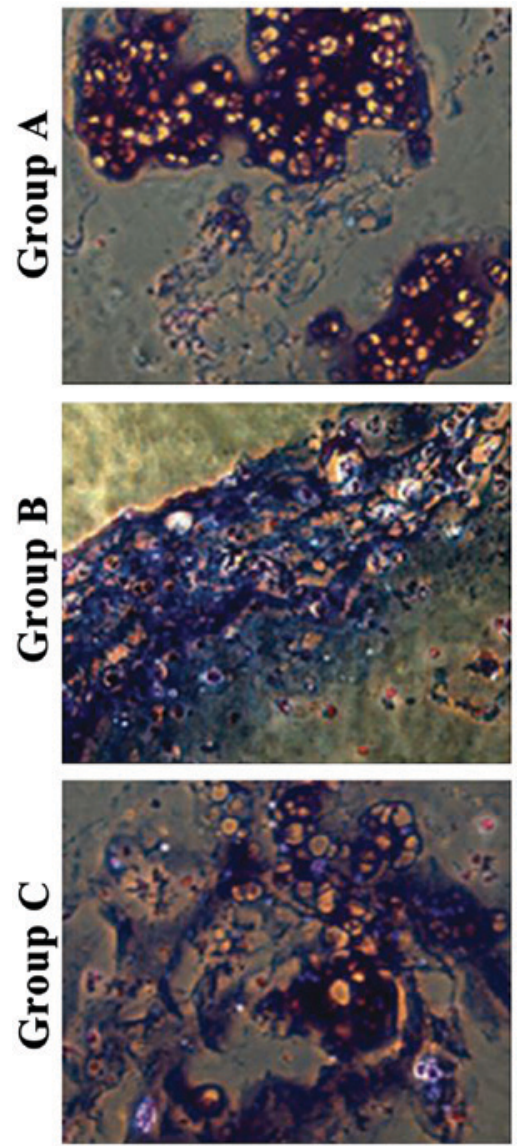

Figure 4. Toluidine blue staining and observation of collagen hydrogelchondrocyte complexes after in vitro culture for 2 weeks in the three groups. Chondrocytes had areas of aggregation, and some were stained purple-red in group A. Fewer chondrocytes were aggregated with different staining around them in group B, and the aggregation of chondrocytes was not obvious. However, the distribution of chondrocytes was uniform with different staining in group C (x200).

Histological observation. H\&E staining showed that chondrocytes showed obvious aggregation in group A, partial proliferation and aggregation in group $\mathrm{B}$, and uniform distribution in group C (Fig. 3).

Toluidine blue staining showed that chondrocytes in group A had aggregation areas and some were stained purplered. In group B, fewer chondrocytes were aggregated and had different staining color and intensity around them, and aggregation was not obvious. In group $\mathrm{C}$, the distribution of chondrocytes was uniform with different staining (Fig. 4).

Comparison of the relative levels of polymerized proteoglycan $m R N A$ in each group. The relative levels of polymerized proteoglycan mRNA were $0.936 \pm 0.178$ in group A, $0.925 \pm 0.136$ in group $B$, and $0.917 \pm 0.135$ in group $C$. There were no significant differences in the relative levels of polymerized proteoglycan mRNA between the three groups ( $\mathrm{P}>0.05)$ (Fig. 5).

Comparison of the relative levels of type-II collagen $m R N A$ in each group. The relative levels of type-II collagen mRNA were $0.772 \pm 0.201$ in group A, $0.741 \pm 0.183$ in group B, and $0.752 \pm 0.165$ in group $C$. There were no significant differences in the relative levels of type-II collagen mRNA between the three groups ( $\mathrm{P}>0.05)$ (Fig. 6). 


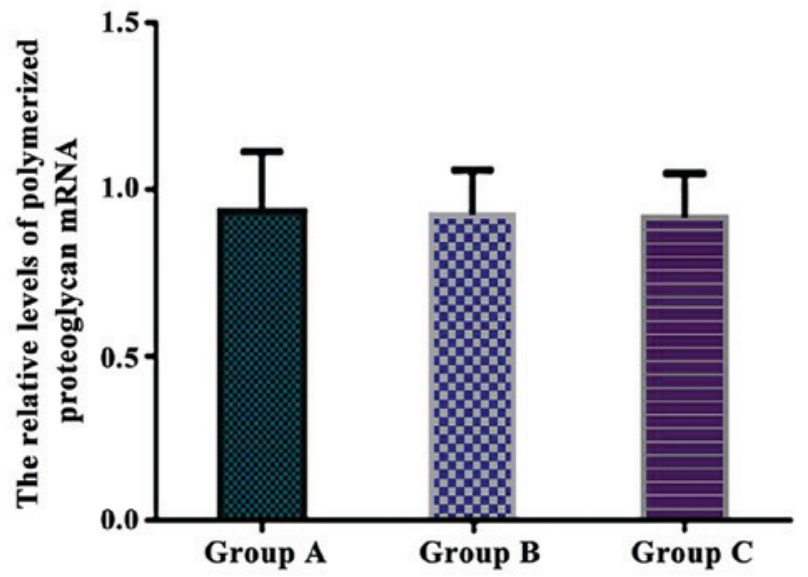

Figure 5. Comparison of the relative levels of polymerized proteoglycan mRNA in each group. Real-time quantitative RT-PCR showed that there were no significant differences in the relative levels of polymerized proteoglycan mRNA between the three groups.

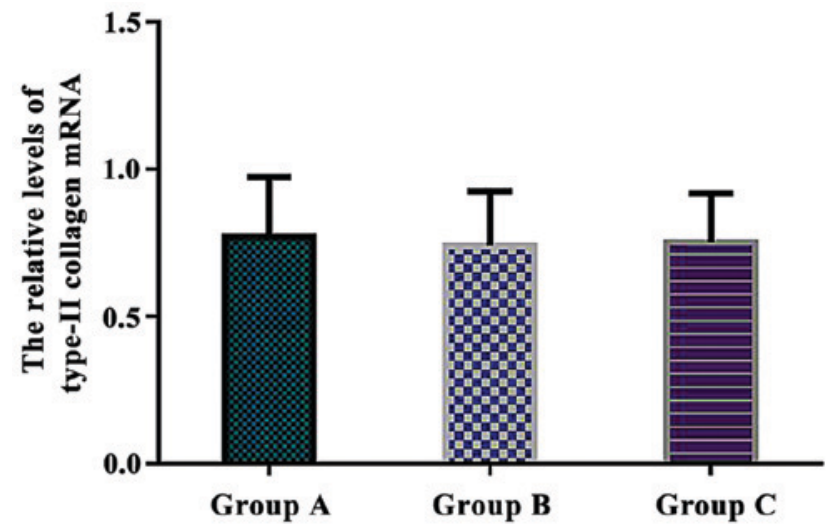

Figure 6. Comparison of the relative levels of type-II collagen mRNA in each group. Real-time quantitative RT-PCR showed that there were no significant differences in the relative levels of type-II collagen mRNA between the three groups $(\mathrm{P}>0.05)$.

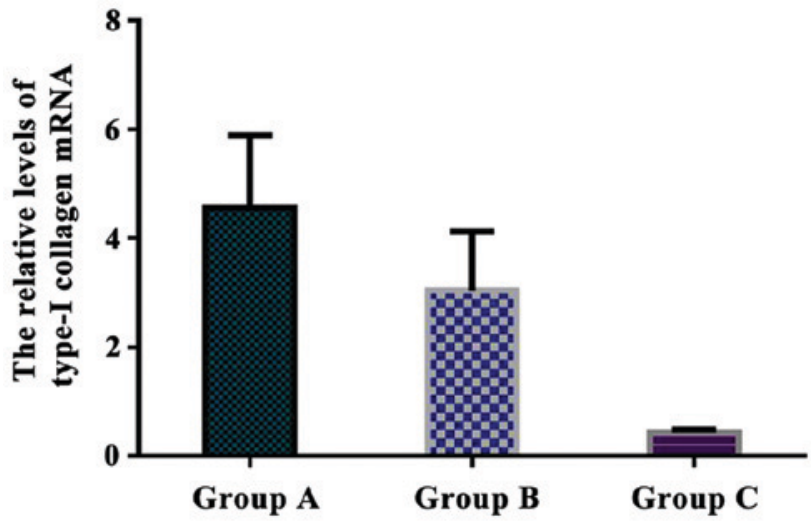

Figure 7. Comparison of the relative levels of type-I collagen mRNA in each group. Real-time quantitative RT-PCR showed that the relative expression of type-I collagen mRNA in group A was significantly higher than that in group $\mathrm{B}$ and $\mathrm{C}(\mathrm{P}<0.05)$. There was a significant difference in type-I collagen mRNA expression between group $\mathrm{B}$ and $\mathrm{C}(\mathrm{P}<0.05)$.

Comparison of the relative levels of type-I collagen $m R N A$ in each group. The relative levels of type-I collagen mRNA

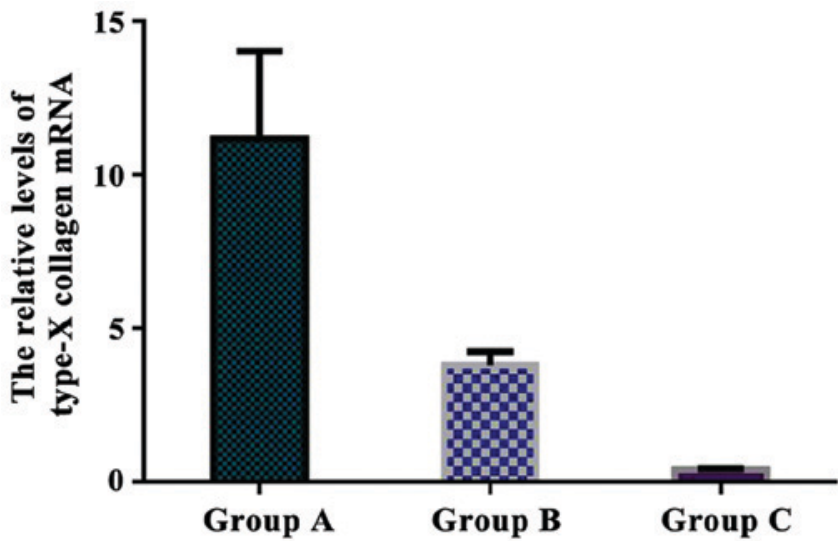

Figure 8. Comparison of the relative levels of type-X collagen mRNA in each group. Real-time quantitative RT-PCR showed that the relative expression of type-X collagen mRNA in group A was significantly higher than that in group $\mathrm{B}$ and $\mathrm{C}(\mathrm{P}<0.05)$. There was a significant difference in type- $\mathrm{X}$ collagen mRNA expression between group $\mathrm{B}$ and $\mathrm{C}(\mathrm{P}<0.05)$.

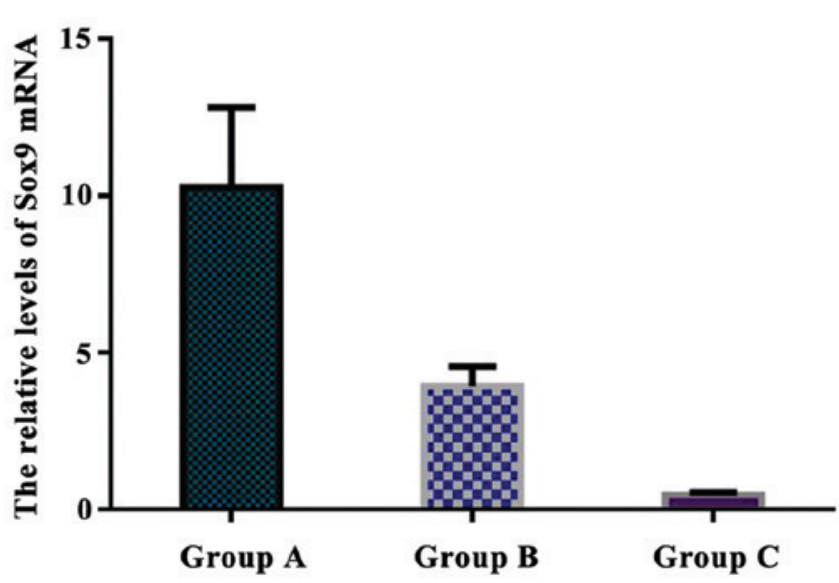

Figure 9. Comparison of the relative levels of Sox9 mRNA in each group. Real-time quantitative RT-PCR showed that the relative expression of Sox 9 mRNA in group A was significantly higher than that in group B and $\mathrm{C}(\mathrm{P}<0.05)$. There was a significant difference in Sox 9 mRNA expression between group $\mathrm{B}$ and $\mathrm{C}(\mathrm{P}<0.05)$.

were $4.561 \pm 1.327$ in group A, 3.046 \pm 1.075 in group B, and $0.432 \pm 0.058$ in group $\mathrm{C}$. The relative expression of type-I collagen mRNA in group A was significantly higher than that in group $\mathrm{B}$ and $\mathrm{C}(\mathrm{P}<0.05)$. Additionally, there was a significant difference in type-I collagen mRNA expression between group B and $\mathrm{C}(\mathrm{P}<0.05)$ (Fig. 7).

Comparison of the relative levels of type-X collagen $m R N A$ in each group. The relative levels of type-X collagen mRNA were $11.172 \pm 2.845$ in group $A, 3.801 \pm 0.446$ in group $B$, and $0.403 \pm 0.027$ in group $C$. The relative expression of type-X collagen mRNA in group A was significantly higher than that in group $\mathrm{B}$ and $\mathrm{C}(\mathrm{P}<0.05)$. There was a significant difference in type-X collagen expression between group $\mathrm{B}$ and $\mathrm{C}$ $(\mathrm{P}<0.05)$ (Fig. 8).

Comparison of the relative levels of Sox $9 \mathrm{mRNA}$ in each group. The relative levels of Sox 9 mRNA were $10.263 \pm 2.551$ in group A, $3.942 \pm 0.628$ in group $\mathrm{B}$, and $0.477 \pm 0.085$ in 
group C. The relative expression of Sox 9 mRNA in group A was significantly higher than that in group $\mathrm{B}$ and $\mathrm{C}(\mathrm{P}<0.05)$. There was a significant difference in Sox 9 mRNA expression between group $\mathrm{B}$ and $\mathrm{C}(\mathrm{P}<0.05)$ (Fig. 9).

\section{Discussion}

Collagen hydrogel is a form of hydrogel that is sensitive to temperature. It is a form of collagen fiber formed by continuous aggregation of collagen molecules of a neutral collagen solution under heating conditions or at $37^{\circ} \mathrm{C}$ (7-9). Related studies showed that (10) collagen concentration is positively correlated with collagen fiber density, which is an important factor for several physical and chemical indexes, such as the mechanical properties and water absorption of collagen gel. Related data showed that (11-13) collagen molecules have biological activity, and can actively participate in signal transduction and cellular behavior at the molecular level. Collagen concentration in the collagen hydrogel therefore plays an important role in its biological function. The biological functions of collagen hydrogels containing different concentrations of collagen may be different $(12,14,15)$. Herein, we aimed to investigate the effects of type-I collagen hydrogel of different concentrations on the growth and differentiation of rabbit chondrocytes.

Related data showed that (16) collagen phenotype plays a highly important role in maintaining the normal structure of cartilage. At different stages of chondrocyte differentiation, the expression of collagen phenotype is different. In the process of differentiation, articular cartilage and epiphyseal cartilage mainly synthesize type-II, VI, IX, and XI collagen, whereas hypertrophic chondrocytes specifically synthesize type-X and II collagen. Type-X and I collagen are synthesized in the process from chondrocyte hypertrophy to differentiation into osteoblast-like cells (17). Type-II collagen and Aggrecan are markers of chondrocytes $(18,19)$. The type-I collagen gene is expressed by fibrous tissues, and can be used to determine whether chondrocytes are differentiated into fibrous tissues (20-22). Sox9 also belongs to the group of chondrocyte fibrosis-related genes, and the type-X collagen gene is an important marker of hypertrophic chondrocytes $(23,24)$. This study showed that the levels of Aggrecan and type-II collagen mRNA in groups $\mathrm{A}, \mathrm{B}$, and $\mathrm{C}$ were not significantly different after in vitro culture for 2 weeks $(\mathrm{P}>0.05)$. The levels of type-I collagen, type-X collagen, and Sox 9 mRNA in group A were significantly higher than those in group B and $\mathrm{C}(\mathrm{P}<0.05)$. The levels of type-I collagen, type- $\mathrm{X}$ collagen, and Sox 9 mRNA were upregulated with increasing concentration of type-I collagen, suggesting that the increase of type-I collagen concentration may induce chondrocyte fibrosis and hypertrophy, thus affecting the formation of normal cartilage tissue. H\&E staining showed that chondrocytes were aggregated significantly in group A, some chondrocytes showed proliferation and aggregation in group B, and the distribution of chondrocytes was relatively uniform in group $\mathrm{C}$, indicating that cell differentiation had occurred.

In conclusion, high concentration of type-I collagen hydrogel can promote chondrocyte fibrosis and upregulation of the expression of type-I collagen, type-X collagen, and Sox 9 mRNA. High concentration type-I collagen hydrogel can result in hypertrophy of articular chondrocytes in a premature manner, which may promote the development of articular cartilage to osteoarthritis.

\section{References}

1. Mahapatra C, Jin GZ and Kim HW: Alginate-hyaluronic acid-collagen composite hydrogel favorable for the culture of chondrocytes and their phenotype maintenance. Tissue Eng Regen Med 13: 538-546, 2016.

2. Foldager CB, Pedersen M, Ringgaard S, Bünger $\mathrm{C}$ and Lind $\mathrm{M}$ : Chondrocyte gene expression is affected by very small iron oxide particles-labeling in long-term in vitro MRI tracking. J Magn Reson Imaging 33: 724-730, 2011.

3. Ge D, Zhang QS, Zabaleta J,Zhang Q, Liu S, Reiser B, Bunnell BA, Braun SE, O'Brien MJ, Savoie FH, et al: Doublecortin may play a role in defining chondrocyte phenotype. Int J Mol Sci 15: 6941-6960, 2014

4. Tsai WB, Chen CH, Chen JF and Chang KY: The effects of types of degradable polymers on porcine chondrocyte adhesion, proliferation and gene expression. J Mater Sci Mater Med 17: 337-343, 2006.

5. Xin W, Heilig J, Paulsson M, Zaucke F and Collagen II: Regulates integrin expression profile and chondrocyte differentiation. Connect Tissue Res 56: 307-314, 2015.

6. Gurusinghe S, Young P, Michelsen J and Strappe P: Suppression of dedifferentiation and hypertrophy in canine chondrocytes through lentiviral vector expression of Sox 9 and induced pluripotency stem cell factors. Biotechnol Lett 37: 1495-1504, 2015.

7. Dou Y, Li N, Zheng Y and Ge Z: Effects of fluctuant magnesium concentration on phenotype of the primary chondrocytes. J Biomed Mater Res A 102: 4455-4463, 2014.

8. Yu L, Ferlin KM, Nguyen BN and Fisher JP: Tubular perfusion system for chondrocyte culture and szp expression. J Biomed Mater Res A 103: 1864-1874, 2015.

9. Ko AR, Huh YH, Lee HC, Song WK, Lee YS and Chun JS: Identification and characterization of arginase II as a chondrocyte phenotype-specific gene. IUBMB Life 58: 597-605, 2006.

10. Duval E, Leclercq S, Elissalde JM, Demoor M, Galéra P and Boumédiene K: Hypoxia-inducible factor 1alpha inhibits the fibroblast-like markers type I and type III collagen during hypoxia-induced chondrocyte redifferentiation: Hypoxia not only induces type II collagen and aggrecan, but it also inhibits type I and type III collagen in the hypoxia-inducible factor lalpha-dependent redifferentiation of chondrocytes. Arthritis Rheum 60: 3038-3048, 2009.

11. Johnson JS, Morscher MA, Jones KC, Moen SM, Klonk CJ, Jacquet R and Landis WJ: Gene expression differences between ruptured anterior cruciate ligaments in young male and female subjects. J Bone Joint Surg Am 97: 71-79, 2015.

12. Kontturi LS, Järvinen E, Muhonen V, Collin EC, Pandit AS, Kiviranta I, Yliperttula M and Urtti A: An injectable, in situ forming type II collagen/hyaluronic acid hydrogel vehicle for chondrocyte delivery in cartilage tissue engineering. Drug Deliv Transl Res 4: 149-158, 2014.

13. Heo J, Koh RH, Shim W, Kim HD, Yim HG and Hwang NS: Riboflavin-induced photo-crosslinking of collagen hydrogel and its application in meniscus tissue engineering. Drug Deliv Transl Res 6: 148-158, 2016.

14. Park H, Guo X, Temenoff JS, Tabata Y, Caplan AI, Kasper FK and Mikos AG: Effect of swelling ratio of injectable hydrogel composites on chondrogenic differentiation of encapsulated rabbit marrow mesenchymal stem cells in vitro. Biomacromolecules 10: 541-546, 2009.

15. Lien SM, Ko LY and Huang TJ: Effect of pore size on ECM secretion and cell growth in gelatin scaffold for articular cartilage tissue engineering. Acta Biomater 5: 670-679, 2009.

16. Galván JA, García-Martínez J, Vázquez-Villa F, García-Ocaña M, García-Pravia C, Menéndez-Rodríguez P, González-del Rey C, Barneo-Serra L and de los Toyos JR: Validation of COL11A1/ procollagen 11A1 expression in TGF- $\beta 1$-activated immortalised human mesenchymal cells and in stromal cells of human colon adenocarcinoma. BMC Cancer 14: 867, 2014.

17. Faikrua A, Wittaya-areekul S, Oonkhanond B and Viyoch J: A thermosensitive chitosan/corn starch/ $\beta$-glycerol phosphate hydrogel containing TGF- $\beta 1$ promotes differentiation of MSCs into chondrocyte-like cells. Tissue Eng Regen Med 11: 355-361, 2014. 
18. Jin R, Moreira Teixeira LS, Krouwels A, Dijkstra PJ, van Blitterswijk CA, Karperien M and Feijen J: Synthesis and characterization of hyaluronic acid-poly(ethylene glycol) hydrogels via Michael addition: An injectable biomaterial for cartilage repair. Acta Biomater 6: 1968-1977, 2010.

19. Schindler OS: Current concepts of articular cartilage repair. Acta Orthop Belg 77: 709-726, 2011.

20. Li P, Wei X, Guan Y, Chen Q, Zhao T, Sun C and Wei L: MicroRNA-1 regulates chondrocyte phenotype by repressing histone deacetylase 4 during growth plate development. FASEB J 28: 3930-3941, 2014.

21. Tew SR, Li Y, Pothacharoen P, Tweats LM, Hawkins RE and Hardingham TE: Retroviral transduction with SOX9 enhances re-expression of the chondrocyte phenotype in passaged osteoarthritic human articular chondrocytes. Osteoarthritis Cartilage 13 80-89, 2005.
22. Darling EM and Athanasiou KA: Retaining zonal chondrocyte phenotype by means of novel growth environments. Tissue Eng 11: 395-403, 2005.

23. Zhang $M$ and Wang J: Epigenetic regulation of gene expression in osteoarthritis. Genes Dis 2: 69-75, 2015.

24. Balakrishnan B and Banerjee R: Biopolymer-based hydrogels for cartilage tissue engineering. Chem Rev 111: 4453-4474, 2011. 